\title{
Sensitivity of Ixodes ricinus (L., 1758) and Dermacentor reticulatus (Fabr., 1794) ticks to entomopathogenic fungi isolates: preliminary study
}

\author{
Anna Szczepańska ${ }^{1} \cdot$ Dorota Kiewra $^{1} \cdot$ Kinga Plewa-Tutaj $^{1}$ (D) $\cdot$ Dagmara Dyczko $^{1} \cdot$ Katarzyna Guz-Regner $^{2}$
}

Received: 23 October 2019 / Accepted: 6 July 2020 / Published online: 14 July 2020

(C) The Author(s) 2020

\begin{abstract}
Entomopathogenic fungi of the genus Beauveria and Metarhizium play an important role in controlling the population of arthropods. However, the data on their effectiveness against ticks focus mainly on species that do not occur in Europe. The aim of the study was to assess the effectiveness of entomopathogenic fungi against two of the most important tick species in Europe: Ixodes ricinus and Dermacentor reticulatus. In our study, the majority of tested entomopathogenic fungi strains showed potential efficacy against both tick species; however, D. reticulatus was less susceptible in comparison to I. ricinus. The observed mortality of ticks was up to $100 \%$ by using all commercial strains as well as three out of nine of the environmental strains. Among all tested fungi, the most effective against both tick species was environmental strain Metarhizium anisopliae $\mathrm{LO} 4(1)$ with $\mathrm{LC}_{50}$ values: $2.6 \times 10^{3} \mathrm{cfu} / \mathrm{ml}-5.7 \times 10^{5} \mathrm{cfu} / \mathrm{ml}$. Botanigard proved to be more effective than MET52 with $\mathrm{LC}_{50}$ values: $6.8 \times 10^{3} \mathrm{cfu} /$ $\mathrm{ml}-3.3 \times 10^{6} \mathrm{cfu} / \mathrm{ml}$. The conducted bioassays indicate the potential possibility of using the environmental isolates of entomopathogenic fungi, as well as commercial strains in control of local populations of I. ricinus and D. reticulatus; however, the possibility of using them in vivo requires more research.
\end{abstract}

Keywords Ixodes ricinus $\cdot$ Dermacentor reticulatus $\cdot$ Entomopathogenic fungi $\cdot$ Biocontrol

\section{Introduction}

Ticks are one of the most important parasites due to transmission of pathogens of tick-borne diseases (TBD). For limiting TBD cases, the individual prophylaxis is mainly recommended; however, the control of the tick population in the environment is also desirable. Among the natural factors limiting the populations of arthropods, there are microorganisms, including entomopathogenic fungi. However, the data concerning

Section Editor: Charlotte Oskam

Kinga Plewa-Tutaj

kinga.plewa-tutaj@uwr.edu.pl

1 Department of Microbial Ecology and Environmental Protection, Institute of Genetics and Microbiology, University of Wroclaw, Przybyszewskiego str. 63/77, 51-148 Wroclaw, Poland

2 Department of Microbiology, Institute of Genetics and Microbiology, University of Wroclaw, Przybyszewskiego str. 63/77, 51-148 Wroclaw, Poland their effectiveness against ticks are scarce and show that different tick species, even the different developmental stages, differ in sensitivity to the bioagent.

The study on the efficiency of fungi against ticks was focused mainly on the genus Rhipicephalus and additional taxa including Ixodes scapularis, Dermacentor variabilis, and Amblyomma variegatum (Onofre et al. 2001; Kirkland et al. 2004; Tuininga et al. 2009; Hedimbi et al. 2011). The data on the potential use of the entomopathogenic fungi against Ixodes ricinus, the tick of the greatest medical and veterinary importance in Europe, are limited and cover mainly the study on larvae and nymphs of this species and engorged adults (Hartelt et al. 2008; Alekseev 2011; Wassermann et al. 2016; PiraliKheirabadi et al. 2016). So far, there is no published data involving the possibility of using entomopathogenic fungi against Dermacentor reticulatus, an important vector of Babesia canis. However, it is known that in the natural conditions, entomopathogenic fungi can infect both $I$. ricinus and D. reticulatus. Entomopathogenic fungi isolated from these tick species were identified as Beauveria bassiana, Beauveria tenella, Lecanicillium lecanii, Isaria fumosorosea, Isaria farinosa, Verticillium aranearum, Beauveria 
brongniartii, and Beauveria pseudobassiana (Samsinakova et al. 1974; Kalsbeek et al. 1995; Munteanu et al. 2014).

The aims of this study were as follows: (1) to obtain environmental fungi strains with potential entomopathogenic properties; (2) to estimate in vitro the effectiveness of entomopathogenic fungi against the most frequently recorded tick species in Europe, i.e., I. ricinus and D. reticulatus; (3) to compare the effectiveness of environmental isolates with commercial strains.

\section{Material and methods}

\section{Tick collection for bioassay}

Ixodes ricinus and Dermacentor reticulatus ticks were collected using the standard flagging method in Wroclaw agglomeration (Poland). Collected ticks were placed in plastic tubes and kept in the refrigerator until they were used for the bioassay (max. for 1 week). Only adults of $I$. ricinus and D. reticulatus identified according Estrada-Pena et al. (2017) were used for the bioassay.

\section{The isolation of entomopathogenic fungi}

The entomopathogenic fungi were isolated from 38 soil samples, collected from the Osobowicki Forest area (Wroclaw, SW Poland) using the insect bait method (Zimmermann 1986). As per similar properties, larvae of Tenebrio molitor were used (Sharma et al. 2018). Ten larvae were placed in each soil sample dampened with distilled water on Petri dishes (incubation at $22 \pm 1{ }^{\circ} \mathrm{C}, 30$ days in the darkness) and checked daily. Dead larvae were removed to the sterile weighing bottle with $100 \mu$ of the sterile distilled water on the bottom margin of the bottle to keep the humidity. The larvae were observed daily for visible fungal growth. After the spore production, individual fungi strains were isolated on potato dextrose agar (PDA, Biocorp).

\section{Fungi identification}

Fungi strains were inoculated onto $25 \mathrm{ml}$ of the liquid and sterile Sabouraud dextrose agar and incubated on a shaker for 7 days. The mycelium was harvested by mechanical filtration in a Büchner funnel (Whatman no. 1 filter paper), washed with sterile distilled water, then frozen at $20{ }^{\circ} \mathrm{C}$, and stored until required. The DNA extraction was carried out using the GenoPlast Biochemicals isolation kit. The ITS region was used to amplify the 5.8S rDNA gene of length range between 600 and $700 \mathrm{bp}$. The ITS region of isolated species was amplified using the universal primer set ITS4 (5' TCCTCCGCTTATTG ATATGC 3') and ITS5 (5' GGAAGTAAAAGTCGTAACAA GG 3') (Pérez-González et al. 2014). Positive amplifications were purified using the DNA purification kit (GenoPlast Biochemicals) and then sent for sequencing (Genomed, Warsaw). The sequencing results were compared with the sequences listed in the National Center for Biotechnology Information (https://blast.ncbi.nlm.nih.gov/Blast.cgi).

\section{Preparation of a fungal spore suspension}

Eight selected environmental soil isolates were used for the bioassay: 5 strains of Metarhizium anisopliae LO4(1), LO10(1), LO52(1), LO52(2), LO47(3), 1 strain of Metarhizium robertsii LO26(2), 1 strain of Isaria fumosorosea LO34(3), 1 strain of Beauveria bassiana

Table 1 Lethal concentrations $\left(\mathrm{LC}_{50}\right)$ of Ixodes ricinus and Dermacentor reticulatus infected with different fungal strains

\begin{tabular}{|c|c|c|c|c|c|}
\hline \multirow[t]{2}{*}{ Strain } & \multirow[t]{2}{*}{ Germination (\%) } & \multicolumn{4}{|l|}{$\mathrm{LC}_{50}(\mathrm{cfu} / \mathrm{ml})$} \\
\hline & & I. ricinus (†) & I. ricinus $($ Љ) & D. reticulatus $($ () & D. reticulatus (ठิ) \\
\hline Botanigard & 97 & $6.8 \times 10^{3}$ & $3.3 \times 10^{6}$ & $5.9 \times 10^{5}$ & $5.9 \times 10^{5}$ \\
\hline MET52 & 95 & $1.6 \times 10^{6}$ & $9.5 \times 10^{4}$ & $2.0 \times 10^{6}$ & $3.9 \times 10^{5}$ \\
\hline I. fumosorosea LO34(3) & 96 & $1.2 \times 10^{8}$ & $1.9 \times 10^{9}$ & $1.0 \times 10^{5}$ & $1.5 \times 10^{14}$ \\
\hline M. robertsii LO26(2) & 92 & $3.9 \times 10^{6}$ & $2.7 \times 10^{5}$ & $3.3 \times 10^{6}$ & $4.6 \times 10^{6}$ \\
\hline B. bassiana LO37(1) & 97 & $5.6 \times 10^{6}$ & $3.4 \times 10^{6}$ & $1.5 \times 10^{7}$ & $5.6 \times 10^{7}$ \\
\hline M. anisopliae LO10(1) & 91 & $8.7 \times 10^{3}$ & $3.8 \times 10^{3}$ & $5.5 \times 10^{4}$ & $4.4 \times 10^{7}$ \\
\hline M. anisopliae LO52(1) & 92 & $2.9 \times 10^{7}$ & $2.9 \times 10^{8}$ & $1.2 \times 10^{7}$ & $6.0 \times 10^{7}$ \\
\hline M. anisopliae LO52(2) & 95 & $1.4 \times 10^{10}$ & n.e. & $5.0 \times 10^{6}$ & n.e. \\
\hline M. anisopliae LO4(1) & 95 & $2.6 \times 10^{3}$ & $5.2 \times 10^{4}$ & $1.0 \times 10^{4}$ & $5.7 \times 10^{5}$ \\
\hline B. bassiana IGM & 90 & $5.2 \times 10^{7}$ & $9.8 \times 10^{8}$ & $8.1 \times 10^{8}$ & $5.6 \times 10^{6}$ \\
\hline M. anisopliae LO47(3) & 98 & $1.0 \times 10^{7}$ & $1.2 \times 10^{7}$ & n.e. & n.e. \\
\hline
\end{tabular}

n.e. not effective 
LO37(1), and additionally one strain B. bassiana IGM1 from the microorganism collection of the Institute of Genetics and Microbiology, University of Wroclaw, isolated from a housefly (Musca domestica). Bioassays were also carried out using two commercial strains: $M$. anisopliae (MET52 Granular, Lot: 1511MG09) and B. bassiana (22WP Botanigard, Borregaard, BioPlant, Lot: 22WP141002). All fungi strains were cultured on PDA medium $\left(22^{\circ} \mathrm{C}, 3\right.$ weeks $)$. Mature colonies were harvested to $0.1 \%$ Tween 80 and centrifuged (4000 rpm, $5 \mathrm{~min}$ ) to separate the spores from the hyphae. The spore concentration was determined using the Fuchs-Rosenthal chamber.

\section{Sporulation test and bioassay}

Before the bioassay, a spore germination test was performed. The $1 \mathrm{ml}$ of prepared suspension was incubated on PDA medium (room temperature, $18 \mathrm{~h}$ ). The ratio of germinating to non-germinating spores was counted according to formula: $\% \mathrm{~s}=(\mathrm{gs} / \mathrm{ngs}) \times 100(\%)$, where gs - number of germinating spores and ngs - number of non-germinating spores. Strains with $>90 \%$ of germinating spores were used in bioassays.

Ten individual unfed ticks separately for each tick species (D. reticulatus/I. ricinus) and sex (female/male) were used for each fungal dilution and the control (Hartelt et al. 2008; PiraliKheirabadi et al. 2016). Ticks were washed in the sterile saline solution, dried, and immersed in the fungal solution for $3 \mathrm{~min}$. At least two different suspensions of the fungal conidia were used. The suspension concentration ranged between $10^{2}$ and $10^{8} \mathrm{cfu} / \mathrm{ml}$. To each tested fungi strain, the control group treated with a saline solution was conducted simultaneously. After the immersion, ticks were transferred to the sterile containers with tissue paper moistened with sterile distilled water to keep $80 \%$ of the relative humidity and kept in the darkness at a temperature of $23^{\circ} \mathrm{C}$. Mortality observations were made daily over 3 weeks, and the paralysis of ticks (straightened legs, no response to $\mathrm{CO}_{2}$ stimuli) was recognized as the lethal effect.

\section{Statistical analyses}

The data on the effects of the fungi on ticks was presented as a dosage causing the death of $50 \%$ of the population $\left(\mathrm{LC}_{50}\right)$. The $\mathrm{LC}_{50}$ values were calculated using the Finney (1952) probit analysis method with the $\mathrm{LC}_{50} / \mathrm{LD}_{50}$ calculator. The tool is designed to calculate the dosages with the Abbot's correction.

\section{Results and discussion}

Obtained fungi strains, with potential entomopathogenic properties from soil, were classified into 4 species: Metarhizium anisopliae, Metarhizium robertsii, Isaria fumosorosea, and Beauveria bassiana. The results of bioassay showed the difference in the potential of tick control, between the tested fungi strains and the tick species (Table 1; Fig. 1). However, the majority of our environmental isolates of fungi and commercial strains showed potential efficacy against both Ixodes ricinus and Dermacentor reticulatus. The observed mortality of unfed ticks was up to $100 \%$ using both commercial strains (Botanigard, MET52) as well as three environmental strains: B. bassiana LO37(1), M. anisopliae LO10(1), and $M$. anisopliae LO4(1). We found D. reticulatus to be less susceptible to entomopathogenic fungi than I. ricinus. Males of both tick species were much less susceptible compared to females. The most effective against both tick species was M. anisopliae LO4(1) with $\mathrm{LC}_{50}$ for $I$. ricinus of $2.6 \times$ $10^{3} \mathrm{cfu} / \mathrm{ml}$ (females) to $5.2 \times 10^{4} \mathrm{cfu} / \mathrm{ml}$ (males) and for

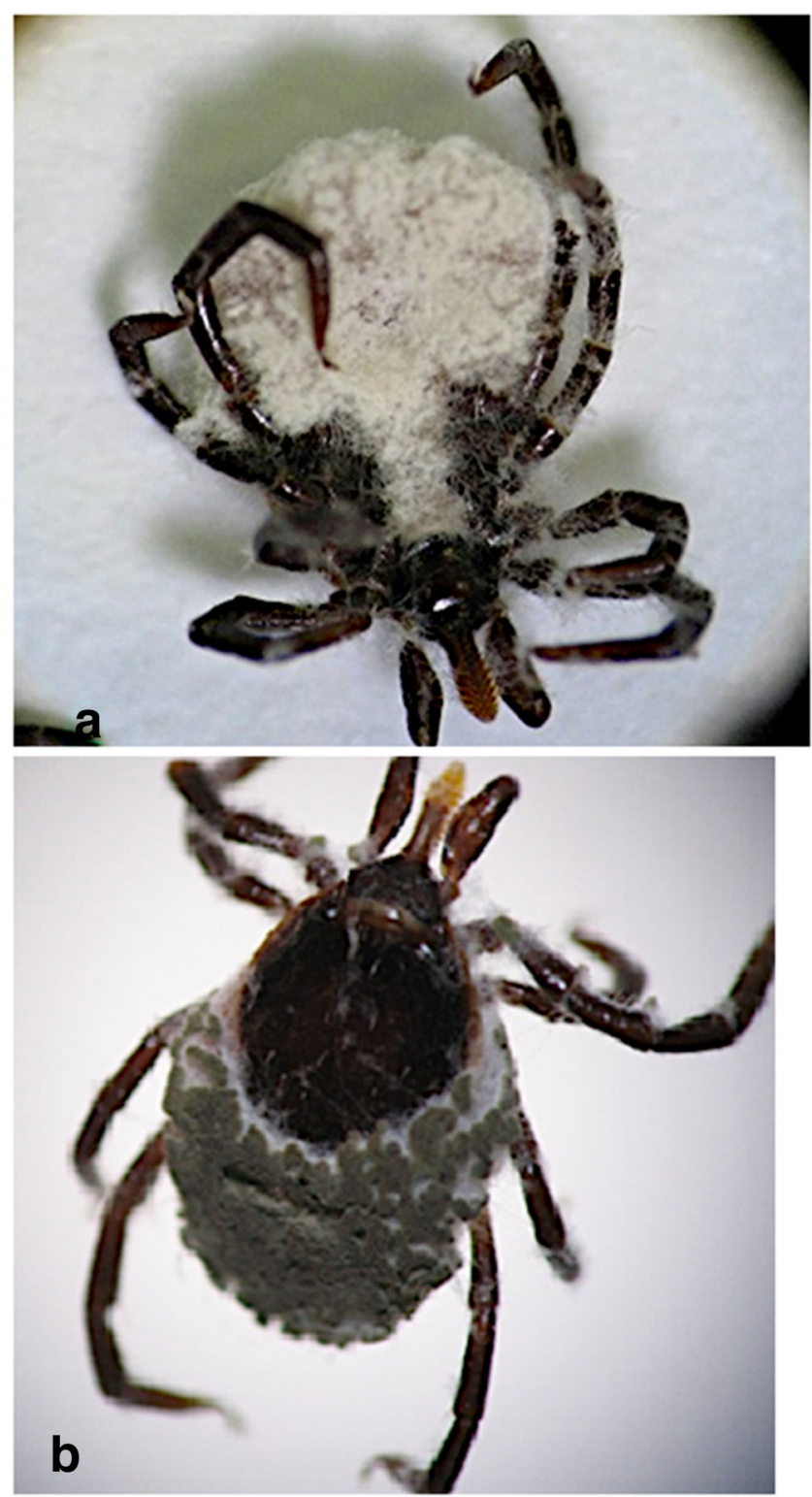

Fig. 1 Fungal mycelia of M. anisopliae (Met52) growth on the cuticle of I. ricinus female. A Ventral side. b Dorsal side 
D. reticulatus of $1.0 \times 10^{4} \mathrm{cfu} / \mathrm{ml}$ (females) to $5.7 \times 10^{5} \mathrm{cfu} / \mathrm{ml}$ (males). The efficacy of environmental fungi strains, including M. anisopliae, was also confirmed in the case of immature and engorged I. ricinus. Hartelt et al. (2008) observed limiting the number of nymphs to $80 \%$ after 30 days. Pirali-Kheirabadi et al. (2016) obtained up to $100 \%$ mortality against populations of engorged females of $I$. ricinus after using $M$. anisopliae IRAN $437 \mathrm{C}$ at a concentration of $2.4 \times 10^{7}$ spores $/ \mathrm{ml}$.

In our study, the tick of both species was less susceptible to $B$. bassiana comparing to M. anisopliae. B. bassiana is also effective against I. scapularis and Rhipicephalus sanguineus and less effective against $D$. variabilis (Kirkland et al. 2004). Our research found I. fumosorosea as the least effective fungi species against ticks. Low mortality with using I. fumosorosea was also observed for $R$. sanguineus larvae $(<10 \%$ mortality after 7 days) and Rhipicephalus microplus larvae (5.5\% mortality after 10 days) (Samish et al. 2001; Angelo et al. 2012). Thus, I. fumosorosea turned out to be the least useful in tick control.

Among commercial strains, Botanigard proved to be more effective than MET52 with $\mathrm{LC}_{50}$ ranging between $6.8 \times$ $10^{3} \mathrm{cfu} / \mathrm{ml}$ for $I$. ricinus females (Botanigard) and $2.0 \times$ $10^{6} \mathrm{cfu} / \mathrm{ml}$ for $D$. reticulatus females (MET52). The virulence of Botanigard was also confirmed for the ticks of Hyalomma lusitanicum, and Amblyomma americanum limiting the average number of tick species in the environment by $90 \%$, and on the host (Oryctolagus cuniculus) up to $80 \%$ (Cradock and Needham 2011; González et al. 2016).

Our research shows that several fungi strains can be considered as candidates for the biological control of I. ricinus and D. reticulatus ticks. However, further investigations, including in vivo tests, are required. Among local environmental entomopathogenic fungi tested in our study, the most promising for the biological control of I. ricinus and D. reticulatus turned out to be M. anisopliae strain LO4(1) as well as commercial products Botanigard and MET52.

Funding information Open Access funding provided by University of Wroclaw.

\section{Compliance with ethical standards}

Conflict of interest The authors declare that they have no competing interests.

Open Access This article is licensed under a Creative Commons Attribution 4.0 International License, which permits use, sharing, adaptation, distribution and reproduction in any medium or format, as long as you give appropriate credit to the original author(s) and the source, provide a link to the Creative Commons licence, and indicate if changes were made. The images or other third party material in this article are included in the article's Creative Commons licence, unless indicated otherwise in a credit line to the material. If material is not included in the article's Creative Commons licence and your intended use is not permitted by statutory regulation or exceeds the permitted use, you will need to obtain permission directly from the copyright holder. To view a copy of this licence, visit http://creativecommons.org/licenses/by/4.0/.

\section{References}

Alekseev AN (2011) Environmentally safe control of ticks: use of Ixodes (Acarina, Ixodidae) tick sexual behavior peculiarities for pathogenic fungal effect reinforcement. Int J Acarol 37:156-165

Angelo IC, Fernandes EK, Bahiense TC, Perinotto WM, Golo PS, Moraes AP, Bittencourt VR (2012) Virulence of Isaria sp. and Purpureocillium lilacinum to Rhipicephalus microplus tick under laboratory conditions. Parasitol Res 111:1473-1480

Cradock KR, Needham GR (2011) Beauveria bassiana (Ascomycota: Hypocreales) as a management agent for free-living Amblyomma americanum (Acari: Ixodidae) in Ohio. Exp Appl Acarol 53:5762. https://doi.org/10.1007/s10493-010-9381-9

Estrada-Pena A, Mihalca AD, Patney TN (2017) Ticks of Europe and North Africa. A guide to species identification. Springer

Finney DJ (1952) Probit analysis (2nd Ed). J Inst Actuaries 78:388-390

González J, Valcárcel F, Pérez-Sánchez JL, Tercero-Jaime JM, Cutuli MT, Olmeda AS (2016) Control of Hyalomma lusitanicum (Acari: Ixodidade) ticks infesting Oryctolagus cuniculus (Lagomorpha: Leporidae) using the entomopathogenic fungus Beauveria bassiana (Hyocreales: Clavicipitaceae) in field conditions. J Med Entomol 53:1396-1402. https://doi.org/10.1093/jme/tjw088

Hartelt K, Wurst E, Collatz J, Zimmermann G, Kleespies RG, Oeheme RM, Kimmig P, Steidle JLM, Mackenstedt U (2008) Biological control of the tick Ixodes ricinus with entomopathogenic fungi and nematodes: preliminary results from laboratory experiments. Int $\mathbf{J}$ Food Microbiol 298:314-320

Hedimbi M, Kaaya GP, Chinsembu KC (2011) Mortalities induced by entomopathogenic fungus Metarhizium anisopliae to different ticks of economic importance using two formulations. Int Res J Microbiol 2:141-145

Kalsbeek V, Frandsen F, Steenberg T (1995) Entomopathogenic fungi associated with Ixodes ricinus ticks. Exp Appl Acarol 19:45-51

Kirkland BH, Westwood GS, Keyhani NO (2004) Pathogenicity of entomopathogenic fungi Beauveria bassiana and Metarhizium anisopliae to Ixodidae tick species Dermacentor variabilis, Rhipicephalus sanguineus, and Ixodes scapularis. J Med Entomol 41:705-711

Munteanu NV, Mitkovets PV, Mitina GV, Movila A, Tokarev YS, Leclerque A (2014) Prevalence of Beauveria pseudobassiana among entomopathogenic fungi isolated from the hard tick, Ixodes ricinus. Ticks Tick Borne Dis 5:641-648. https://doi.org/10.1016/j. ttbdis.2014.04.015

Onofre SB, Miniuk CM, de Barros NM, Azevedo JL (2001) Pathogenicity of four strains of entomopathogenic fungi against the bovine tick Boophilus microplus. Am J Vet Res 62:1478-1480

Pérez-González VH, Guzmán-Franco AW, Alatorre-Rosas R, HernándezLópez J, Hernández-López A, Carrillo-Benítez MG, Baverstock J (2014) Specific diversity of the entomopathogenic fungi Beauveria and Metarhizium in Mexican agricultural soils. J Invertebr Pathol 119: 54-61. https://doi.org/10.1016/j.jip.2014.04.004

Pirali-Kheirabadi KH, Razzaghi-Abyaneh M, Eslamifar A, Halajian A, Nabian S (2016) Scanning electron microscopy (SEM) analysis and biological control of Ixodes ricinus using entomopathogenic fungi. Mycol Iran 3:39-46. https://doi.org/10.22043/mi.2017.41288.1065

Samish M, Gindin G, Alekseev E, Glazer I (2001) Pathogenicity of entomopathogenic fungi to different developmental stages of Rhipicephalus sanguineus (Acari: Ixodidae). J Parasitol 87:13551359

Samsinakova A, Kalalova S, Daniel M, Dusbabek F, Honzakova E, Cerny V (1974) Entomogenous fungi associated with the tick Ixodes ricinus (L.). Folia Parasit 21:39-48

Sharma L, Oliveira I, Torres L, Marques G (2018) Entomopathogenic fungi in Portuguese vineyards soils: suggesting a 'GalleriaTenebrio-bait method' as bait-insects Galleria and Tenebrio 
significantly underestimate the respective recoveries of Metarhizium (robertsii) and Beauveria (bassiana). MycoKeys 38:1-23

Tuininga AR, Miller JL, Morath SU, Daniels TJ, Falco RC, Marchese M, Sahabi S, Rosa D (2009) Isolation of entomopathogenic fungi from soils and Ixodes scapularis (Acari: Ixodidae) ticks: prevalence and methods. J Med Entomol 46:557-565

Wassermann M, Selzer P, Steidle JLM, Mackenstedt U (2016) Biological control of Ixodes ricinus larvae and nymphs with Metarhizium anisopliae blastospores. Ticks Tick Borne Dis 7:768-771
Zimmermann G (1986) The 'Galleria bait method' for detection of entomopathogenic fungi in soil. J Appl Entomol 102:213-215

Publisher's note Springer Nature remains neutral with regard to jurisdictional claims in published maps and institutional affiliations. 